\title{
The variability of cosmic methanol masers in massive star-forming regions
}

\author{
Jabulani P. Maswanganye and Michael J. Gaylard \\ HartRAO, P O BOX 443, Krugersdorp 1740, South Africa \\ email: jabulani@hartrao.ac.za, mike@hartrao.ac.za
}

\begin{abstract}
The methanol masers associated with G35.20-1.74 were monitored at $12178 \mathrm{MHz}$ for four years and $6668 \mathrm{MHz}$ for five years using the $26 \mathrm{~m}$ Hartebeesthoek telescope. This source showed irregular variability and a single large flare event during the monitoring window.
\end{abstract}

Keywords. masers, radio lines: ISM, ISM: molecules, stars: formation.

\section{Introduction and Observations}

Class II methanol masers (MMII) are associated with high mass star-forming regions and often appear to be projected on ultra-compact ionised hydrogen (UCHII) regions. The $6668 \mathrm{MHz} J_{k}=5_{1}-6_{0} \mathrm{~A}^{+}$and $12178 \mathrm{MHz} J_{k}=2_{0}-3_{-1}$ E are the brightest class II methanol masers. Hartebeesthoek Radio Astronomy Observatory (HartRAO) monitors samples of methanol masers in the two transitions in a quest to understand the source of the observed variability. G35.20-1.74 (W48, IRAS 18592+0108) is one such maser source, which is associated with a small UCHII region (Wood \& Churchwell, 1989)

The monitoring was carried out using the $26 \mathrm{~m}$ telescope. Both the $6668 \mathrm{MHz}$ and $12178 \mathrm{MHz}$ receivers provide dual left- and right- circular polarisation outputs. Spectra were obtained at half-power beamwidth offsets to measure and correct for pointing errors. Spectra were calibrated using Ott et al. (1994) scale with 3C123 and 3C218 as the primary calibrators.

\section{Results and discussion}

The spectra and time series for G35.20-1.74 at $6668 \mathrm{MHz}$ and $12178 \mathrm{MHz}$ are shown in Figures 1 and 2 respectively. The variations appear to occur randomly, but are correlated (i) in many of the peaks in each transition and (ii) in peaks at similar velocities in the two transitions. The large flare at about MJD 54172 is notable. The $42.663 \mathrm{~km} . \mathrm{s}^{-1}$ time series at $12178 \mathrm{MHz}$ and $42.780 \mathrm{~km} . \mathrm{s}^{-1}$ at $6668 \mathrm{MHz}$ are atypical in showing flare onset changing to a sharp decrease to a short duration minimum. The $46.205 \mathrm{~km} . \mathrm{s}^{-1}$ time series at $6668 \mathrm{MHz}$ shows an exponential decay with e-folding time of 361 days. Similar behaviour was noted in a maser peak in G188.95+0.89 by van der Walt (2011).

The maser spots in this source were modelled as lying in a rotating Keplerian disk by Minier et al. (2000). Referring to the maser spot map in their Fig. 3, the separation of the extreme North and South maser spots in the map is $1300 \mathrm{AU}=7.5$ light days (also interpreted as the disk diameter) and 3.2 days for West-East separation. The maximum of the large flare occurred earliest in masers at velocities around $41 \mathrm{~km} . \mathrm{s}^{-1}$ (Southern spot group) and 44.5 to $45.3 \mathrm{~km} . \mathrm{s}^{-1}$ (Northern group); then after 12 - 15 days at 42.3 $42.8 \mathrm{~km} . \mathrm{s}^{-1}$ (West central group), then after another $\sim 15$ days at $43.5-44 \mathrm{~km} . \mathrm{s}^{-1}$ (East central group). 


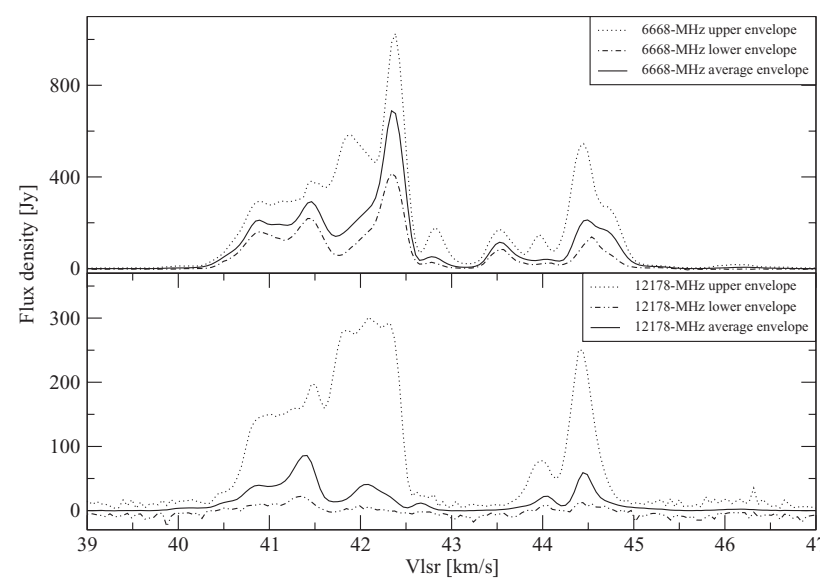

Figure 1. Methanol spectra for G35.20-1.74 at $6668 \mathrm{MHz}$ and $12178 \mathrm{MHz}$.
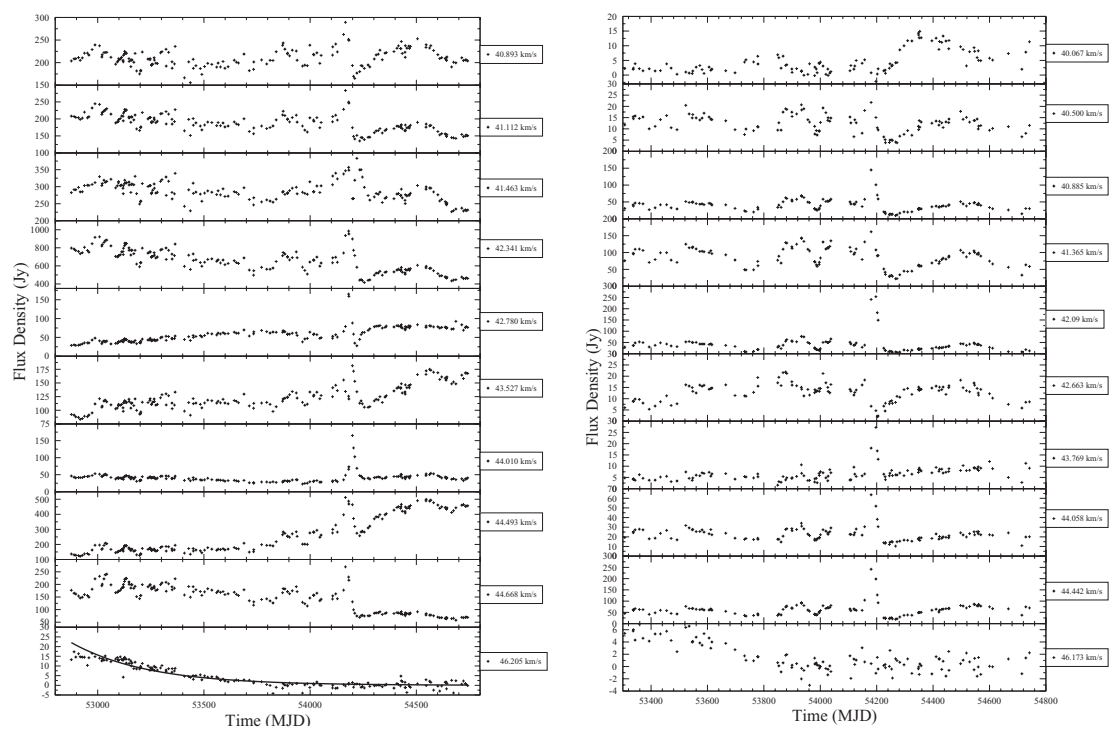

Figure 2. Time series for G35.20-1.74 at $6668 \mathrm{MHz}$ (left panel) and $12178 \mathrm{MHz}$ (right panel).

By contrast, the correlated random variations before the large flare, e.g. from MJD 53800 to 54100 , show little or no time delay in the different time series. This suggests the flare and the other random variations have different origins. The correlated random variations may represent a response to infrared or radio continuum emission changes in the central source, which will produce small time delays, given the extent of masing spots. The much larger delays seen in the large flare could represent a response to a rotating or precessing jet.

\section{References}

Minier, V., Booth, R. S., \& Conway, J. E. 2000, A\&\&A, 362, 1093

Ott, M., Witzel, A., Quirrenbach, A., Krichbaum, T. P., Standke, K. J., Schalinski, C. J., \& Hummel, C. A. 1994, A\& $A, 284,331$

van der Walt, D. J. 2011, $A \mathscr{G} A, 141,152$

Wood, D. O. S. \& Churchwell, E. 1989, ApJS, 69, 831 